[5] J. Max, "Quantizing for minimum distortion," IRE Trans. Inform. Theory, vol. IT-6, pp. 7-12, Jan. 1960.

[6] D. K. Sharma, "Design of absolutely optimal quantizers for a wide class of distortion measures," IEEE Trans. Inform. Theory, vol. IT-24, pp. $693-702$, Nov. 1978

[7] X. Wu, "Algorithmic approaches to quantization," Ph.D. thesis, Univ. of Calgary, 1988.

[8] __, "Optimal bi-level quantization and its application to multilevel quantization," IEEE Trans. Inform. Theory, vol. 37, pp. 160-163, Jan. 1991.

[9] , "On convergence of Lloyd's method I," IEEE Trans. Inform. Theory, vol. 38, pp. 171-174, Jan. 1992.

[10] __ "Efficient algoritlums for discrete optimal quantization," J. Algorithms, vol. 12, no. 4, pp. 663-673, Dec. 1991.

[11] __ "Vector quantizer design by constrained global optimization," Proc. IEEE Data Compression Conf. '92, Mar. 1992, pp. 132-141.

\section{Average Number of Facets per Cell in Tree-Structured Vector Quantizer Partitions}

\author{
Kenneth Zeger and Miriam R. Kantorovitz
}

\begin{abstract}
Upper and lower bounds are derived for the average number of facets per cell in the encoder partition of binary tree-structured vector quantizers. The achievability of the bounds is described as well. It is shown in particular that the average number of facets per cell for unbalanced trees must lie asymptotically between 3 and 4 in $R^{2}$, and each of these bounds can be achieved, whereas for higher dimensions it is shown that an arbitrarily large percentage of the cells can each have a lincar number (in codebook size) of facets. Analogous results are also indicated for balanced trees.
\end{abstract}

Index Terms-Tree-structured vector quantization, data compression, computational geometry.

\section{INTRODUCTION}

A vector quantizer (VQ) $Q: \boldsymbol{R}^{d} \rightarrow \boldsymbol{C}$ is a mapping from $d$ dimensional Euclidean space $\boldsymbol{R}^{d}$ into a finite set of code vectors $\boldsymbol{C} \subset \boldsymbol{R}^{d}$, called a codebook. Every VQ induces a finite partition of $\boldsymbol{R}^{d}$ into sets, $Q^{-1}(y)$, for $y \in C$, called cells, which are the inverse images of vectors in $C$ under the quantizer mapping. In an unstructured VQ, a nearest-neighbor encoder partitions $\boldsymbol{R}^{d}$. into a Voronoi diagram, consisting of a finite set of convex polytopal cells.

A binary tree-structured vector quantizer (TSVQ) $Q$ can formally be defined recursively as any VQ with codebook $C$ that satisfies either of the following two conditions.

1) $|C|=1$.

2) There exists a TSVQ $\hat{Q}$, with codebook $\hat{C}$, and a half-space $H$ such that for some $x, y \in C$ and some $z \in \hat{C}$,

$$
\boldsymbol{C}=(\hat{\boldsymbol{C}} \cup\{x, y\})-\{z\}
$$

Manuscript received Nov. 3, 1991; revised Sept. 8, 1992. This work was supported in part by the National Science Foundation under Grants NCR-9009766 and NCR-91-57 770, and in part by the Hewlett-Packard Co. This work was presented in part at the International Symposium on Information Theory and Its Applications, Ibusuki, Japan, Dec. 1991.

$\mathrm{K}$. Zeger is with the Coordinated Science Laboratory, University of Illinois, 1308 W. Main Street, Urbana, IL 61801.

M. R. Kantorovitz is with the Department of Mathematics, University of Illinois, Urbana, IL 61801.

IEEE Log Number 9207086.

$$
\begin{aligned}
\forall w & \in \boldsymbol{C} \cap \hat{\boldsymbol{C}}, \quad Q^{-1}(w)=\hat{Q}^{-1}(w) \\
Q^{-1}(x) & =\hat{Q}^{-1}(z) \cap H \\
Q^{-1}(y) & =\hat{Q}^{-1}(z) \cap\left(\boldsymbol{R}^{d}-H\right) .
\end{aligned}
$$

Each application of the second condition to an existing TSVQ yiclds a new TSVQ by cutting (or splitting) the cell $\hat{Q}^{-1}(z)$ into two new cells, $Q^{-1}(x)$ and $Q^{-1}(y)$. Such a convex polytopal cell is split by the hyperplane boundary of $H$.

The set of all TSVQ's is built up by recursively applying the previous two conditions. General TSVQ's are referred to as unbalanced. A TSVQ is said to be balanced if either it has exactly one codevector or else it was obtained by successively applying the second TSVQ condition exactly once to every codevector in some existing balanced TSVQ. As in general VQ, TSVQ's also partition $\boldsymbol{R}^{d}$ into a finite set of convex polytopal cells. This follows from the fact that every encoding region is a finite intersection of half-spaces.

It will be assumed throughout that the intersection of any cellsplitting hyperplane with a face of the split cell is of lower dimension then that of the face itself. This implies, for example, that if a cell $S$ is split into two cells with a hyperplane $H$, then $H$ cannot intersect any vertices of $S$ and can only intersect boundary lines of $S$ in single points. This assumption could equivalently have been stated as a general position [1] restriction, corresponding to zero probability events for TSVQ partitions arising from random variables with continuous density functions.

A facet of a convex polytope in $\boldsymbol{R}^{d}$ is any $(d-1)$-dimensional face of the polytope. Two cells in a quantizer partition are neighbors if each has a distinct facet, one of which is a subset of the other. Equivalently, two cells are neighbors if the intersection of their closures has dimension $d-1$. For a VQ encoder partition in general position, there is a one-to-one correspondence between the facets of a cell and the cell's neighbors. However, for TSVQ, it is possible that one cell could be adjacent to several other cells via the same facet; in general, the number of facets per cell is less than or equal to the number of neighbors of the cell. Often, however, these two quantities are very similar or equal. Note also that in general, a TSVQ partition of $\boldsymbol{R}^{d}$ is not a nearest neighbor partition. For a given convex polytopal partition $\Omega$ of $\boldsymbol{R}^{d}$ into $n$ cells, define

1) $F_{d}(n)=$ average number of facets per cell in $\Omega$;

2) $G_{d}(n)=n F_{d}(n)$

3) $M_{d}(n)=$ maximum number of facets of a cell in $\Omega$.

$G_{d}(n)$ is the sum over all cells in $\Omega$ of the number of facets bounding each cell. The results in this paper are stated in terms of bounds on $F_{d}(n)$, whereas many of the proofs are given in terms of $G_{d}(n)$ for convenience.

It is of both theoretical and practical interest to know $F_{d}(n)$ for various applications in quantization theory. One such application arises in multiprocessor design of QV [2], where every pair of neighboring cells is examined in the context of an iterative improvement algorithm. Processor memory and time consumption in this case are proportional to the total number of ccll ncighbor pairs, which is lower bounded by the total number of facets in the encoder partition. In [3], an asymptotic analysis of TSVQ is performed by choosing a model that assumes every cell in a TSVQ encoder partition has exactly $2 d$ facets in $d$-dimensional space. Under this assumption, a useful formula was derived in [3] for the high resolution distortion of a TSVQ, which was supported by experimental evidence.

One might question, in general, whether it is the case that all or most of the cells in a TSVQ have exactly $2 d$ facets, or whether even 
on average this must be true. In fact, it might be questioned whether $F_{d}(n)=O(1)$ can always be guaranteed or whether alternatively $F_{d}(n)$ can be unbounded in $n$.

Note that since every cell of a vector quantizer with $u$ codevectors cannot share more than one facet with any other cell we obtain the trivial upper bound $F_{d}(n) \leq n-1$. Using a construction hased on the existence of simplicial neighborly $d$-polytopes, Klee [4] showed the remarkable result that for $d \geq 4$ this bound is achievable with equality for unstructured vector quantizers whose codevectors are in general position. In such quantizers, every cell is adjacent to every other cell in the encoder partition. In two dimensions, a straightforward application of Euler's theorem for planar graphs shows that $F_{2}(n) \leq 6$. Seidel [5] similarly derived expressions for counting the faces in $d$-dimensional Voronoi diagrams in terms of the number of faces on a polytope of one higher dimension, obtained by stereographic projection.

Several authors have also examined non-Voronoi partitions of $\boldsymbol{R}^{d}$. Using the Euler-Poincare formula from combinatorial topology, Buck [6] determined the number of cells that result from dividing up $R^{d}$ by a given number of arbitrarily placed hyperplanes in general position, and generalized the results to projective $d$-space. In [7] it was shown that if convex polytopes are chosen at random, generated according to some continuous probability distribution, then $F_{d}(n)=2 d$ as $n \rightarrow$ $\infty$. Many interesting properties of randomly constructed polytopal divisions of $\boldsymbol{R}^{d}$ were given in [8]-[11].

In this correspondence, we derive several bounds on $F_{d}(n)$ for TSVQ and point out the achievability of these. Specifically, it is shown that for two-dimensional unbalanced TSVQ, the average number of facets per cell is asymptotically bounded above by 4 and below by 3 , and that the bounds are achievable. For higher dimensional spaces, an upper bound of $n / 2$ and a lower bound of 3 are given. It is also shown that $n / 4$ and 3 respectively are achievable in this case. At present, it is an open question as to whether the $n / 2$ bound is achievable. In $\boldsymbol{R}$, it is trivially always the case that $F_{1}(n)=2-2 / n$.

In $\boldsymbol{R}^{2}$, it is shown that if an asymptotically large fraction of the TSVQ cells are bounded, then $F_{2}(n) \approx 4$. This would lend some support to the assumption made in [3] that $F_{d}(n)=2 d$ for the case $d=2$. However, for $d>2$, this might not be the case. It is shown analogously that for balanced TSVQ with $d>2$ the upper bound on the average number of facets per cell is reduced to $\log _{2} n$. It should be emphasized, though, that the achievability of the bounds presented are best and worst cases, over the class of all TSVQ's, and it is a question for future study as to how likely they are to occur for various practical TSVQ systems.

\section{BOUNDS ON NUMBER OF FACETS PER CELL FOR UNBALANCED TREES}

The encoder partition of an unbalanced TSVQ can be viewed as being formed via a sequence of cuts by hyperplanes. The following facts will prove useful in the various proofs.

Fact 1) Each cut increases the number of cells by exactly one. After $n-1$ cuts there are precisely $n$ cells.

Fact 2) If the hyperplane forming the $n$th cut of the TSVQ intersects exactly $m$ facets of the cell it splits, then the total number of facets in the partition increases by $m+2$.

Fact 3) For $n>1$, the $n$th cut introduces at least 3, but no more than $M_{d}(n)+2$ new facets.

Fact 4) After $n$ cuts, no cell can have more than $n$ facets, i.c., for all $n, M_{d}(n) \leq n-1$.

Proposition 1: For unbalanced TSVQ, the average number of facets per cell satisfies

$$
\begin{array}{lll}
3-4 / n \leq F_{d}(n) \leq n / 2+1 / 2 & d>2, & n \geq 1 \\
3-4 / n \leq F_{d}(n) \leq 4-7 / n & d=2, & n \geq 3
\end{array}
$$

Proof: Consider the two cases separately.

Case 1: $d>2$.

It is proven by induction on $n$ that

$$
3 n-4 \leq G_{d}(n) \leq n(n+1) / 2 .
$$

There are no facets until at least two cells exist, so the basis step is satisfied, since $G_{d}(1)=0$. Now suppose the inequality in (1) holds up to $n$, and consider the case for $n+1$. Then, we get

$$
\begin{aligned}
G_{d}(n+1) & \leq G_{d}(n)+M_{d}(n)+2 \\
& \leq G_{d}(n)+n+1 \\
& \leq n(n+1) / 2+n+1 \\
& =(n+1)(n+2) / 2,
\end{aligned}
$$

where the three incqualitics follow respectively from Fact 3, Fact 4, and the induction hypothesis in (1). For the lower bound, note that after there are at least two cells, any new cell splitting increases the total face count by at least three, since at least one face must split into two and the splitting hyperplane is counted as two new facets. This gives

$$
\begin{aligned}
G_{d}(n+1) & \geq G_{d}(n)+3 \\
& \geq(3 n-4)+3 \\
& =3(n+1)-4,
\end{aligned}
$$

the second inequality following from the induction hypothesis.

Case 2: $d=2$.

First, note the fact that $F_{2}(1)=0$ and $F_{2}(2)=1$. Again using induction on $n$ we show that for $n \geq 3$,

$$
3 n-4 \leq G_{2}(n) \leq 4 n-7 \text {. }
$$

The first cut adds two facets to the total, giving $G_{2}(2)=2$, and the second cut adds three facets to the total giving $G_{2}(3)=5$, which satisfies the basis step. Every cut following the first two cuts intersects at most two edges of the cell it splits. From Fact 2 and the induction hypothesis,

$$
G_{d}(n+1) \leq G_{d}(n)+4 \leq 4 n-7+4=4(n+1)-7 .
$$

For the lower bound, use the same argument as for $d>2$.

The next several results exhibit the bounds' achievability.

Proposition 2: For every $d>2$ and $n>1$ there exists an unbalanced TSVQ such that

$$
F_{d}(n) \geq n / 4
$$

Proposition 3: For $d=2$ and every $n>2$ there exists an unbalanced TSVQ such that

$$
F_{d}(n)=4-7 / n
$$

Proposition 4: For every $d \geq 2$ and $n>1$ there exists an unbalanced TSVQ such that

$$
F_{d}(n)=3-4 / n \text {. }
$$


Proof of Proposition 2: First, note that $G_{d}(2)=2$ and $G_{d}(3)=$ 5 . We present a construction in $\boldsymbol{R}^{d}$ of a TSVQ with $n>3$ cells satisfying $F_{d}(n) \geq n / 4$. Let $K$ be any $(d-1)$-dimensional bounded convex polytope with $m(d-2)$-dimensional faces, where $m \leq n$. Let $P_{0}$ be an unbounded convex polytope in $\boldsymbol{R}^{d}$ containing $K$ as one of its facets. Furthermore, suppose, without loss of generality, that $P_{0}$ lies in $K^{+}$, the closure of one of the two half-spaces determined by $K$ ( $K$ is viewed as a hyperplane in $\left.\boldsymbol{R}^{d}\right)$. The existence and construction of such polytopes is described in [12]. Let $H_{1}, \cdots, H_{m}$ be the hyperplanes in $\boldsymbol{R}^{d}$ that are determined by the $m$ facets of $P_{0}$ that intersect $K$. Let $P$ bc the convex hull (unbounded) of $\left(H_{1} \cap K^{+}\right) \cup \cdots \cup\left(H_{m} \cap K^{+}\right) \cup K . P$ has $m+1$ facets, and thus can be obtained as one cell of a particular TSVQ after $m+1$ cuts have been made. One possible sequence of hyperplanes making these $m+1$ cuts is $H_{1}, \cdots, H_{m}, K$. Proposition 1 implies that

$$
G_{d}(m+2) \geq 3(m+2)-4 \text {. }
$$

The next $n-m-2$ cuts are described as follows. Let $\hat{B}$ be a hyperplane lying in $K^{+}$and parallel to $K$, and let $B=\hat{B} \cap P$. Now, let $H$ be any hyperplane through $P$ such that $H \cap K=\phi$ and $H \cap B=\phi$. Then, $H$ intersects each $H_{i}$ for $i=1, \cdots, m$, and splits $P$ into two new convex polytopes, $P_{1}$ and $P_{2} . P_{1}$ has $m+2$ facets ( $K$ is one of them) and $P_{2}$ has $m+1$ facets.

Relabel $P_{2}$ by $P, H \cap P_{2}$ by $K$, and then repeat the process. In the resulting TSVQ, there are $n>3$ cells and

$$
\begin{aligned}
G_{d}(n) & =G_{d}(m+2)+(n-m-2)(m+2) \\
& \geq(n-m+1)(m+2)-4 .
\end{aligned}
$$

Now choose $m=[n / 2]$, the greatest integer less than or equal to $n / 2$. This gives

$$
\begin{aligned}
G_{d}(n) & \geq(n-n / 2+1)(n / 2-1 / 2+2)-4 \\
& =\frac{n^{2}}{4}+\frac{5}{4} n-\frac{5}{2} \\
& \geq \frac{n^{2}}{4} .
\end{aligned}
$$

Thus, $F_{d}(n) \geq n / 4$.

Proof of Proposition 3: After the first two cuts one has $G_{d}(3)=$ 5 . The third cut can be used to form a triangle, and it intersects two edges, so $G_{d}(4)=G_{d}(3)+4=9$. For the remaining cuts, always choose a bounded cell to cut; in this case, since the cells are convex, the cut must intersect exactly two edges. So from Fact 2 ,

$$
G_{d}(n)=5+(n-3) 4=4 n-7 .
$$

Proof of Proposition 4: The lower bound can be achieved by creating a TSVO with only unbounded cells. Each new cut introduces three facets to the total number of facets and $G_{d}(2)=2$. Thus, $G_{d}=3 n-4$, which can easily be proven by induction.

The following corollary to Proposition 2 shows that there exist $d$-dimensional TSVQ's such that an arbitrarily large fraction of the cells each have a linear number (in codebook size) of facets.

Corollary 1: For $d>2, n>1$, and for every $\alpha \in(0,1)$, there exists a TSVQ with $n$ cells such that at least $\alpha n$ of the cells each have at least $(1-\alpha) n$ facets.

Proof: In the proof of Proposition 2, take $m=[n(1-\alpha)]-2$. In this case, the last $n-m-2$ cuts yield $n-m-2$ cells, each having $[(1-\alpha) n]$ facets. This completes the proof, since $n-m-2 \geq n-n(1-\alpha)=n \alpha$.

\section{Bounds FOR BALANCED TREes}

For balanced trees similar results are obtained, though with a reduction from linear to logarithmic bounds. The results are stated in terms of the number of cells, $n$, in the TSVQ, though it should be remembered that balanced trees only exist when $n$ is some integer power of 2 . In the following proposition, the achievability of the lower bound for $d \geq 2$ and the upper bound for $d=2$ are analogous to the unbalanced case. However, it is unknown at present whether the upper bound $\log _{2} n$ is achievable; in fact it is unknown whether, for a fixed $d>2$, it is possible to exhibit balanced TSVQ's such that $F_{d}(n)$ is unbounded.

Corollary 2 : For balanced TSVQ,

$$
\begin{aligned}
& 3-4 / n \leq F_{d}(n) \leq \log _{2} n, \quad d>2, \quad n>0, \\
& 3-4 / n \leq F_{d}(n) \leq 4-8 / n, \quad d=2, \quad n>0 \text {. }
\end{aligned}
$$

Proof: For $d \geq 2$, the lower bound is proven exactly as in Proposition 1 , by creating only unbounded cells at each stage of splitting. For $d=2$, the upper bound is obtained in the same manner as for unbalanced trees, where at least one new bounded cell is created from each cell splitting, starting with $n=8$, since for $n=1,2,4$, it is only possible to form unbounded cells. This accounts for the slightly different bound than for unbalanced trees. For $d>2$, to obtain the upper bound we use $M_{d}\left(2^{n}\right) \leq n$ and the fact that each of the cells split can introduce no more than $M_{d}(n)+2$ new facets, to get

$$
\begin{aligned}
G_{d}(2 n) & \leq G_{d}(n)+n\left(M_{d}(n)+2\right) \\
& \leq G_{d}(n)+n \log _{2} n+2 n
\end{aligned}
$$

or equivalently after dividing by $2 n$,

$$
F_{d}(2 n) \leq \frac{1}{2} F_{d}(n)+\frac{1}{2} \log _{2} n+1 .
$$

From this recursive inequality it easily follows by induction that $F_{d}(n) \leq \log _{2} n$.

\section{REFERENCES}

[1] F. P. Preparata and M. I. Shamos, Computational Geometry: An Introduction. New York: Springer-Verlag, 1985.

[2] K. Zeger and A. Gersho, "A parallel processing algorithm for vector quantizer design based on subpartitioning," presented at Proc. IEEE Int. Conf. Acoust., Speech, and Signal Processing (ICASSP), Toronto, Canada, May 1991.

[3] D. Neuhoff and D. Lee, "On the performance of tree-structured vector quantization," presented at IEEE Int. Symp. Inform. Theory, Budapest, Hungary, June 24-28, 1991.

[4] V. Klee, "On the complexity of $d$-dimensional Voronoi diagrams,"Archiv Der Mathematik, vol. 34, pp. 75-80, 1980.

[5] R. Seidel, "The complexity of Voronoi diagrams in higher dimensions," Proc. 20th Allerton Conf. Commun., Contr., Comput., Allerton, IL, 1982.

[6] R.C. Buck, "Partition of space," Amer. Math. Monthly, vol. 50, pp. 541-544, 1943.

[7] May and Smith, "Random polytopes: Their definition, generation and aggregate properties," Math. Programming, vol. 24, pp. 39-54, Sept. 1982.

[8] Goudsmit, "Random distribution of lines in a plane," Rev. Modern Phys., vol. 17, no. 2, pp. 321-322, Apr.-July 1945.

[9] R.E. Miles, "Random polygons determined by random lines in a plane," Proc. Nat. Acad. Sci., vol. 52, 1964, pr. 901-907.

[10] __ "Random polygons determined by random lines in a plane II," Proc. Nat. Acad. Sci., vol. 52, 1964, pp. 1157-1160.

[11] P. I. Richards, "Averages for polygons formed by random lincs," Proc. Nat. Acad. Sci., vol. 52, 1964, pp. 1160-1164.

[12] B. Grunbaum, Convex Polytopes. New York: Wiley Interscience, 1967. 